\title{
Comparison of full and partial coverage crowns with CAD/CAM leucite reinforced ceramic blocks on fracture resistance and fractographic analysis
}

\author{
Carlos A. JURADOํ․ Firas MOURAD², Daniel Alberto CORTES TREVINO3, Diogo N. GOUVEIA ${ }^{3}$, Jared HYER ${ }^{4}$, \\ Amira ELGREATLY ${ }^{5}$, Ahmed M. MAHROUS ${ }^{6}$, Franklin GARCIA-GODOY ${ }^{7}$ and Akimasa TSUJIMOTO ${ }^{5}$ \\ ${ }_{1}^{1}$ Texas Tech University Health Sciences Center El Paso, Woody L. Hunt School of Dental Medicine, 5001 El Paso Drive, El Paso, TX 79905, USA \\ ${ }^{2}$ Department of Care Planning and Restorative Sciences, University of Mississippi Medical Center School of Dentistry, 2500 North State Street, \\ Jackson, MS 39216, USA \\ ${ }^{3}$ Department of Restorative and Prosthetic Dentistry, Ohio State University College of Dentistry, 305 W. 12th Avenue, Columbus, OH 43210, USA \\ ${ }^{4}$ A. T. Still University Arizona School of Dentistry \& Oral Health, 5850 E Still Circle Mesa, AZ 85206, USA \\ ${ }^{5}$ Department of Operative Dentistry, University of lowa College of Dentistry, 801 Newton Road, lowa City, IA 52242, USA \\ ${ }^{6}$ Department of Prosthodontics, University of lowa College of Dentistry, 801 Newton Road, lowa City, IA 52242, USA \\ ${ }^{7}$ Department of Bioscience Research, College of Dentistry, University of Tennessee Health Science Center, Memphis Tennessee, USA and Forsyth \\ Institute, Cambridge, MA, USA \\ Corresponding author, Akimasa TSUJIMOTO; E-mail: akimasa-tsujimoto@uiowa.edu
}

\begin{abstract}
To compare full and partial coverage crowns made with different CAD/CAM leucite reinforced ceramic blocks on fracture resistance and fractographic analysis. Full coverage and partial coverage crowns with finish line at $2 \mathrm{~mm}$ and $4 \mathrm{~mm}$ above the gingiva for molars made with IPS Empress CAD and Rosetta BM. After fatigue process, the fracture test and fractographic analysis were conducted. Although the fracture resistance of IPS Empress crowns did not show any statistical differences regardless of the design, both the partial coverage crowns fabricated using Rosetta BM showed significantly higher load at break and peak load than the full coverage crown. The crowns made with IPS Empress showed significantly higher fracture resistance than that made with Rosetta $\mathrm{BM}$, regardless of the restorative design. The fracture resistance and fractographic analysis of CAD/CAM leucite reinforced full and partial coverage crowns were influenced by the restorative design of and material type.
\end{abstract}

Keywords: CAD/CAM, Leucite, Crown, Fracture resistance, Fractographic analysis

\section{INTRODUCTION}

Tooth wear results in loss of occlusal tooth structure and a full coverage crown is the conventional means of oral rehabilitation ${ }^{1)}$. Unfortunately, this restorative approach entails the further removal of tooth structure of teeth already weakened due to wear and multifactorial issues ${ }^{2}$. Currently, more conservative partial coverage crowns represent an accepted treatment option in situations of occlusal wear due to erosion or abrasion ${ }^{3)}$. The designs of partial coverage crowns vary in the location of the finish line of the tooth preparation, with a tendency to place it as supragingival as possible to maintain sound tooth substrates ${ }^{4}$.

With computer-aided design and computer-aided manufacturing $(\mathrm{CAD} / \mathrm{CAM})$ in dentistry, the production of $\mathrm{CAD} / \mathrm{CAM}$ high strength ceramic restorations has become a reality, and increasingly popular in the clinic $^{5)}$. Most CAD/CAM high strength ceramic restorative materials are fabricated in a pre-sintered state that facilitates the milling process and maximizes the lifespan of the burs performing the subtractive processes $^{6)}$.

Currently, leucite and lithium disilicate reinforced ceramic materials are available on the market. Leucite reinforced ceramic materials have generally higher translucency ${ }^{7)}$, weaker strength ${ }^{8)}$ and lower price than lithium disilicate reinforced ceramic materials, and contain $35-45 \%$ by volume of crystals ranging in size from 1 to $5 \mu \mathrm{m}^{9}$. Leucite reinforced pressable ceramic materials were introduced as IPS Empress (Ivoclar Vivadent, Schaan, Liechtenstein) in 1986, and the $\mathrm{CAD} / \mathrm{CAM}$ version was then released as blocks for the CEREC inLab System as IPS ProCAD (Ivoclar Vivadent) in 199810). A previous study reported that material properties CAD/CAM version have shown similar results to the pressable equivalent ${ }^{11)}$, and a 5 year prospective study of partial coverage crowns using IPS ProCAD for molars found a success rate of $97 \%{ }^{12)}$. In 2006 , the properties of the material were improved and it was renamed IPS Empress CAD (Ivoclar Vivadent). The newer CAD/CAM version of the material offered flexural strength of 151.0 MPa, which is comparable to $\mathrm{CAD} / \mathrm{CAM}$ composite blocks, but with a flexural modulus of $32.3 \mathrm{GPa}$, which is much higher ${ }^{13)}$.

With the development of more silica and oxide ceramics such as lithium disilicate and zirconia, the popularity of leucite reinforced ceramic materials has recently decreased ${ }^{10)}$. However, a new type of leucite reinforced ceramic block for $\mathrm{CAD} / \mathrm{CAM}$ restorations, exemplified by Rosetta BM (Bio Hass, Gangwon-do, Korea) has been developed. A previous study reported that this novel leucite reinforced ceramic block has improved properties in comparison to the initial version $^{14)}$. Unfortunately, there are no independent studies evaluating differently designed crowns for 
molars using leucite reinforced ceramic blocks fabricated by different companies.

The purpose of this study was to investigate the comparison of full and partial coverage crowns with different $\mathrm{CAD} / \mathrm{CAM}$ leucite reinforced ceramic blocks on fracture resistance and fractographic analysis. The null hypotheses were that (1) different leucite ceramic blocks would provide the same fracture resistance for each type of crown; (2) partial coverage crowns with different locations of finish line would have the same fracture resistance as full coverage crowns; and (3) partial coverage crowns with different locations of finish line would have the same fracture resistance.

\section{MATERIALS AND METHODS}

Study design of fracture strength measurement Typodont teeth (1560 Dentoform, Columbia Dentoform, Lancaster, PA, USA), lower right first molars, were designed in three different shapes: (1) a full coverage crown preparation; (2) a partial coverage crown preparation with finish line at $2.0 \mathrm{~mm}$; and (3) at 4.0 $\mathrm{mm}$ above the gingiva (Fig. 1). The typodont teeth were digitally imaged and designed with a chairside intraoral scanner and built-in system (Primescan, Dentsply Sirona, Charlotte, NC, USA). Two leucite reinforced ceramic blocks: (1) IPS Empress CAD; and (2) Rosetta BM were tested in this study. Sixty crowns were milled with a chair-side milling machine (MCXL, Dentsply Sirona), ten for each group of the three types of crowns and the two brands of leucite reinforced ceramic block. The milled crowns were polished, glazed and crystalized following the manufacturers' instructions.

The typodont teeth were digitally imaged and designed with a dental 3D scanner (Degree of Freedom HD, DOF, Seoul, Korea) for printing. Sixty matching teeth were printed using a model resin (Formlabs, Somerville, MA, USA) with a desktop dental 3D printer (FormLab $3 \mathrm{~B}$, Formlabs). The crowns were cemented to printed abutments as follows: (1) the crowns were ultrasonically cleaned, water-rinsed and air-dried; (2) the intaglios of the crowns were treated $\mathrm{u}$ sing $5 \%$ hydrofluoric acid agent (IPS Ceramic Etching Gel, Ivoclar Vivadent) for $60 \mathrm{~s}$ and water-rinsed; (3) the crowns were treated using primer (Monobond Plus, Ivoclar Vivadent) and airdried; (4) the crowns were cemented to the printed teeth with dual-cure resin luting cement (Multilink Automix, Ivoclar Vivadent) and light-cured using a light curing unit (Elipar 2500, 3M Oral Care, St. Paul, MN, USA).

The bonded crowns subjected $100 \mathrm{~N}$ load force for 1 million cycles at $1 \mathrm{~Hz}$ in a custom fatigue cycler. Specimens were secured with a steel jig in a vertical position and loaded against a polyoxymethylene ball (Delrin $6.28 \mathrm{~mm}$, Dupont, Wilmington, DE, USA) used to make contact with the occlusal surface of the restoration. At the end of the fatigue test, the crowns were examined to determine if any catastrophic failure had occurred. The crowns that survived the fatigue process were secured on a steel jig and then loaded to fracture in compression in a universal testing machine (Instron 4204, Norwood, MA, USA). A tapered cone-shaped (3.2 $\mathrm{mm}$ at the tip) applicator was placed vertically along the central groove of the crown, with a $1 \mathrm{~mm} / \mathrm{min}$ compressive load rate.

Throughout the fracture test, the load at break (the loading force at which the material cracks), peak load (the loading force at which the material fractures), and the elapsed times until fracture were documented.

\section{Fractographic analysis}

Fractographic analysis of two specimens of each the crowns on the $3 \mathrm{D}$ printed teeth were conducted using scanning electron microscope (ERA 8800FE, Elionix, Tokyo, Japan). The specimens were sputter coated with a thin layer of gold (Quick Coater Type SC-701, Sanyu Electron, Tokyo, Japan). The fractured surfaces of the crowns on the printed teeth were observed with an accelerating voltage at $15 \mathrm{kV}$. a

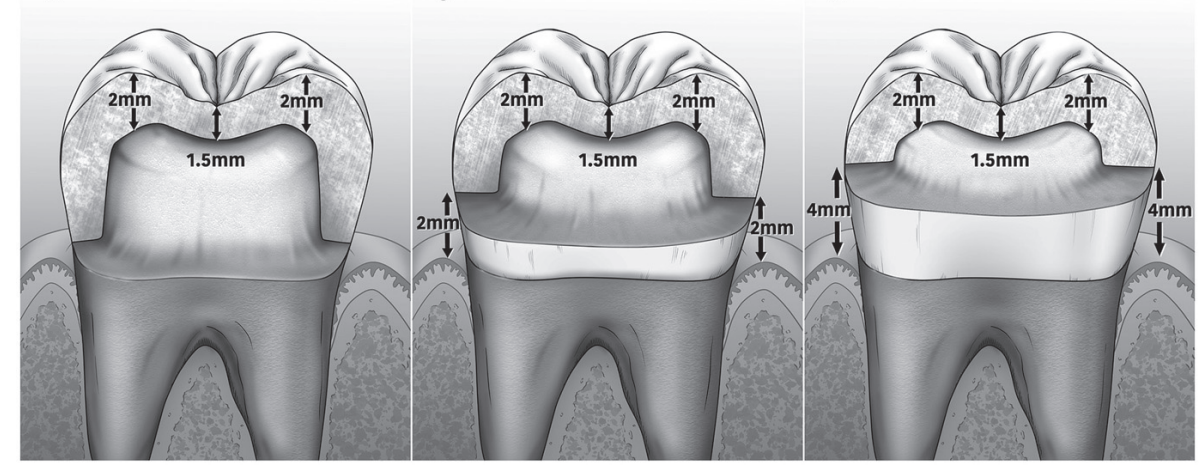

Fig. 1 Types of restorations on a mandibular first molar; (a) full coverage crown; (b) partial coverage crown with finish line at $2 \mathrm{~mm}$; and (c) that at $4 \mathrm{~mm}$ above the gingiva. 


\section{Statistical analysis}

The sample size was determined using the effect size $=0.25$ (medium) or 0.5 (large), $\mathrm{a}=0.05$, power $=0.8$, and number of groups $=6$. The results indicate that a total of 211 samples (medium effect size) or 58 (large effect size) were needed for the fracture loading tests. The analysis showed that 9.7-35.2 samples were needed for each group for the test, and therefore using 10 samples/group, which is within the results of the G-power calculation, was considered appropriate.

Two-way analysis of variance with a Tukey post hoc honest significant difference test was carried out using a software (SPSS Statistics 25, IBM, Armonk, NY, USA) to analyze the gathered data for the influence of (1) material type and (2) restorative design, at a significance level of $5 \%$. The statistical consaltations and analysis were conducted by Dr. Curt Bay at A.T. Still University Arizona School of Dentistry \& Oralhealth.

\section{RESULTS}

\section{Fracture resistance}

The fracture resistance of the CAD/CAM leucite reinforced full and partial coverage crowns with different material and restorative designs on mandibular first molars are shown in Table 1 . The load at break and the peak load were influenced by material type (load at break: $p=0.000$; peak load: $p=0.000)$ and the restorative design (load at break: $p=0.021$; peak load: $p=0.042$ ).

The full and partial coverage crowns with IPS Empress CAD showed 1,210.2-1,329.4 $\mathrm{N}$ for the load at break and 1,372.2-1,399.2 $\mathrm{N}$ for the peak load. There were no significant differences between loads at break or peak of the restorations fabricated from IPS Empress CAD, regardless of restorative design. Thus, if IPS Empress CAD is used for molar crowns, the material appears to be acceptable for use in either full or partial coverage crowns.

On the other hand, the load at break (921.4-1,140.6 $\mathrm{N})$ and the peak load (1,073.6-1,172.3 N) for the partial coverage crowns of Rosetta BM were significantly higher than those for full coverage crowns (load at break: $688.2 \mathrm{~N}$; peak load: $778.5 \mathrm{~N}$ ). Significant differences were observed in the load at break between the partial coverage crowns with Rosetta BM with the finish line at $2 \mathrm{~mm}$ and $4 \mathrm{~mm}$ above the gingiva, but not in the peak load. In addition, the load at break and peak load of full and partial coverage crowns of Rosetta BM were significantly lower than those of IPS Empress CAD, except for the load at break of partial coverage crowns with the finish line at $4 \mathrm{~mm}$ above the gingiva.

Some of the samples with partial coverage crowns failed in the exposed base of the resin abutment, rather than in the crown. These samples were excluded from the analysis, as the values bear no obvious relationship to the strength of the crown.

The elapsed time until fracture was different depending on the material type $(p=0.011)$, but not on the restorative design $(p=0.085)$. The elapsed time until fracture was 76.7-113.7 $\mathrm{ms}$ for the crowns with IPS Empress CAD and 63.0-65.4 ms for the crowns with Rosetta BM. However, the only individual difference that was statistically significant was that between 4 mm partial coverage crowns for IPS Empress CAD and Rosetta BM.

\section{Fractographic analysis}

Fractographic images of the crowns on printed teeth are shown in Figs. 2-7. Note that the varying location of the fracture in the crown can obscure the differences between the restorative designs. Fracture surfaces of the partial coverage crowns were much cleaner, more defined and

Table 1 Fracture resistance of the CAD/CAM leucite reinforced full and partial coverage crowns with different material and restorative designs

\begin{tabular}{|c|c|c|c|c|c|c|c|c|}
\hline \multirow{2}{*}{$\begin{array}{c}\text { Restorative } \\
\text { design }\end{array}$} & \multicolumn{2}{|c|}{$\begin{array}{c}\text { Number of } \\
\text { surviving specimens }\end{array}$} & \multicolumn{2}{|c|}{$\begin{array}{l}\text { Loat at break } \\
\qquad(\mathrm{N})\end{array}$} & \multicolumn{2}{|c|}{$\begin{array}{l}\text { Load at fracture } \\
(\mathrm{N})\end{array}$} & \multicolumn{2}{|c|}{$\begin{array}{l}\text { Elapsed time until } \\
\text { fracture (ms) }\end{array}$} \\
\hline & $\begin{array}{l}\text { IPS } \\
\text { Empress } \\
\text { CAD }\end{array}$ & $\begin{array}{l}\text { Rosetta } \\
\text { BM }\end{array}$ & $\begin{array}{l}\text { IPS } \\
\text { Empress } \\
\text { CAD }\end{array}$ & $\begin{array}{l}\text { Rosetta } \\
\text { BM }\end{array}$ & $\begin{array}{l}\text { IPS } \\
\text { Empress } \\
\text { CAD }\end{array}$ & $\begin{array}{l}\text { Rosetta } \\
\text { BM }\end{array}$ & $\begin{array}{l}\text { IPS } \\
\text { Empress } \\
\text { CAD }\end{array}$ & $\begin{array}{c}\text { Rosetta } \\
\text { BM }\end{array}$ \\
\hline $\begin{array}{l}\text { Full coverage } \\
\text { crowns }\end{array}$ & 10 & 10 & $\begin{array}{l}1,277.9 \\
(316.9)^{\mathrm{a}, \mathrm{A}}\end{array}$ & $\begin{array}{c}688.2 \\
(135.1)^{\mathrm{a}, \mathrm{B}}\end{array}$ & $\begin{array}{l}1,372.2 \\
(349.5)^{\mathrm{a}, \mathrm{A}}\end{array}$ & $\begin{array}{c}778.50 \\
(146.8)^{\mathrm{a}, \mathrm{B}}\end{array}$ & $\begin{array}{c}76.7 \\
(21.9)^{\mathrm{a}, \mathrm{A}}\end{array}$ & $\begin{array}{c}64.4 \\
(17.9)^{\mathrm{a}, \mathrm{A}}\end{array}$ \\
\hline $\begin{array}{l}\text { Partial coverage crowns } \\
\text { with finish line at } 2.0 \\
\mathrm{~mm} \text { above the gingiva }\end{array}$ & 9 & 10 & $\begin{array}{l}1,210.2 \\
(324.2)^{\mathrm{a}, \mathrm{A}}\end{array}$ & $\begin{array}{c}921.4 \\
(64.6)^{\mathrm{b}, \mathrm{B}}\end{array}$ & $\begin{array}{l}1,392.1 \\
(304.7)^{\mathrm{a}, \mathrm{A}}\end{array}$ & $\begin{array}{l}1,073.6 \\
(77.9)^{\mathrm{b}, \mathrm{B}}\end{array}$ & $\begin{array}{c}83.22 \\
(29.65)^{\mathrm{a}, \mathrm{A}}\end{array}$ & $\begin{array}{c}65.4 \\
(10.6)^{\mathrm{a}, \mathrm{A}}\end{array}$ \\
\hline $\begin{array}{l}\text { Partial coverage crowns } \\
\text { with finish line at } 4.0 \\
\mathrm{~mm} \text { above the gingiva }\end{array}$ & 10 & 8 & $\begin{array}{l}1,329.4 \\
(331.6)^{\mathrm{a}, \mathrm{A}}\end{array}$ & $\begin{array}{l}1,140.6 \\
(196.7)^{\mathrm{c}, \mathrm{A}}\end{array}$ & $\begin{array}{l}1,399.2 \\
(299.3)^{\mathrm{a}, \mathrm{A}}\end{array}$ & $\begin{array}{l}1,172.3 \\
(157.2)^{\mathrm{b}, \mathrm{B}}\end{array}$ & $\begin{array}{l}113.70 \\
(46.1)^{\mathrm{a}, \mathrm{A}}\end{array}$ & $\begin{array}{c}63.0 \\
(9.6)^{\mathrm{a}, \mathrm{B}}\end{array}$ \\
\hline
\end{tabular}

Values in parenthesis are standard deviations. The same lowercase letter in the same vertical column indicates no significant difference $(p>0.05)$. The same uppercase letter in the same individual horizontal row indicates no significant difference $(p>0.05)$. 


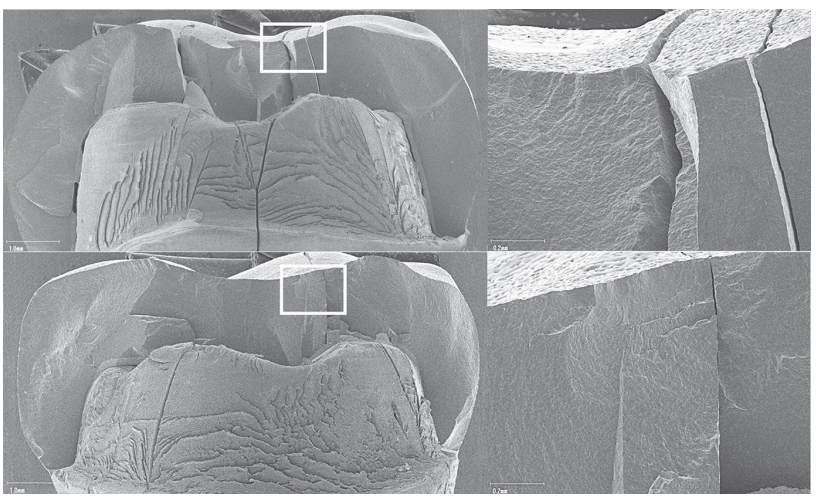

Fig. 2 Fractographic images of full coverage crowns for IPS Empress CAD at $\times 20$ for a and $c$, and $\times 100$ for $\mathrm{b}$ and $\mathrm{d}$ magnification for different specimens.

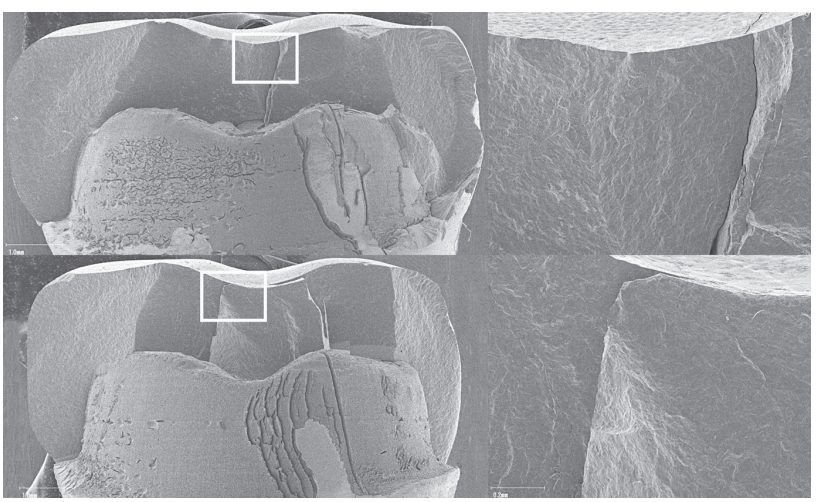

Fig. 3 Fractographic images of partial coverage crowns with finish line at $2 \mathrm{~mm}$ above the gingiva for IPS Empress CAD at $\times 20$ for $a$ and $c$, and $\times 100$ for $b$ and $d$ magnification for different specimens.

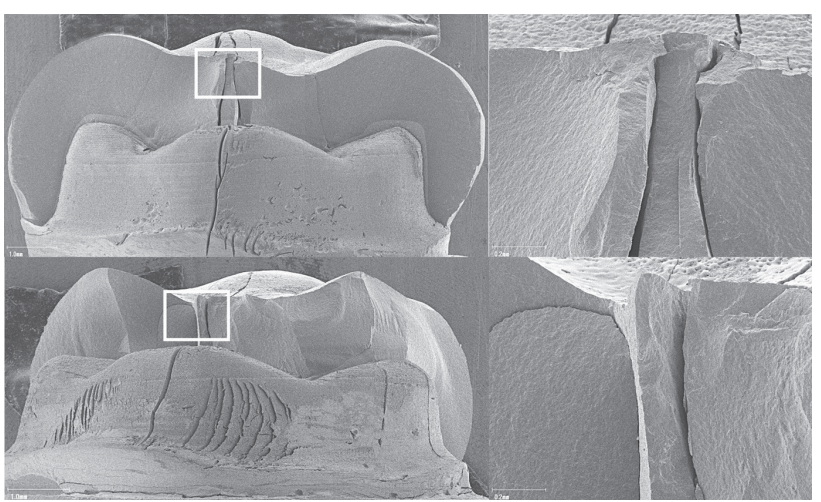

Fig. 4 Fractographic images of partial coverage crowns with finish line at $4 \mathrm{~mm}$ above the gingiva for IPS Empress CAD at $\times 20$ for $a$ and $c$, and $\times 100$ for $b$ and $d$ magnification for different specimens.

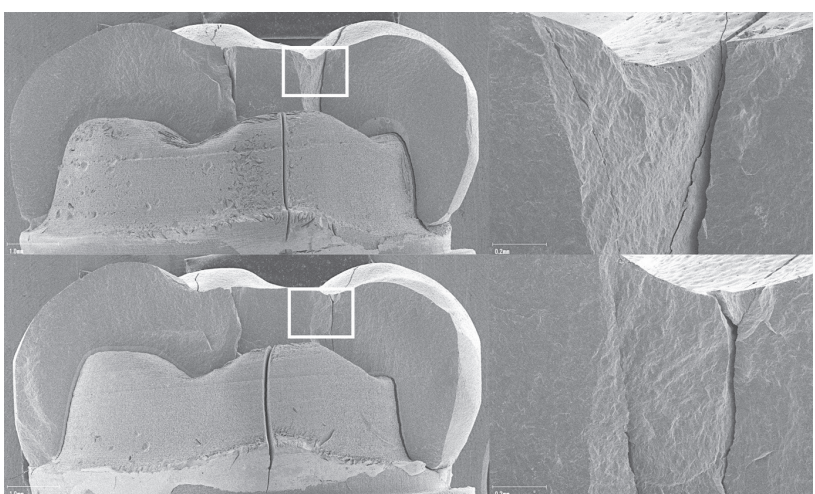

Fig. 5 Fractographic images of full coverage crowns for Rosetta BM at $\times 20$ for a and $c$, and $\times 100$ for $b$ and $\mathrm{d}$ magnification for different specimens.

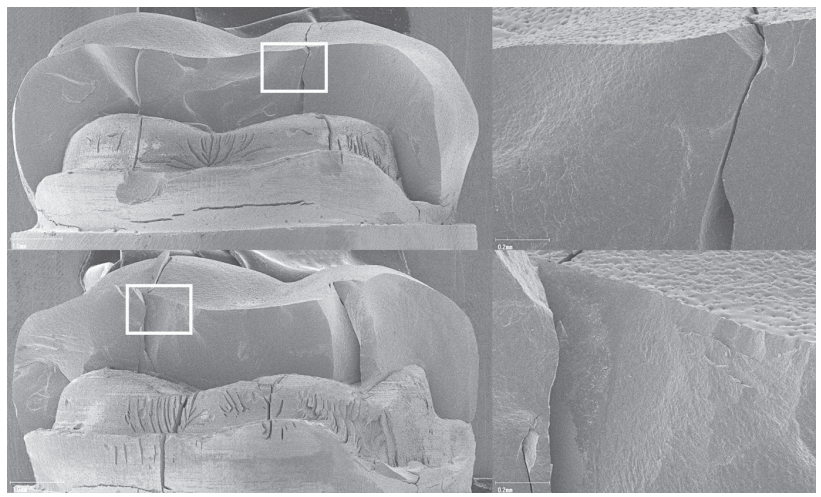

Fig. 6 Fractographic images of partial coverage crowns with finish line at $2 \mathrm{~mm}$ above the gingiva for Rosetta $\mathrm{BM}$ at $\times 20$ for $\mathrm{a}$ and $\mathrm{c}$, and $\times 100$ for $\mathrm{b}$ and $\mathrm{d}$ magnification for different specimens.

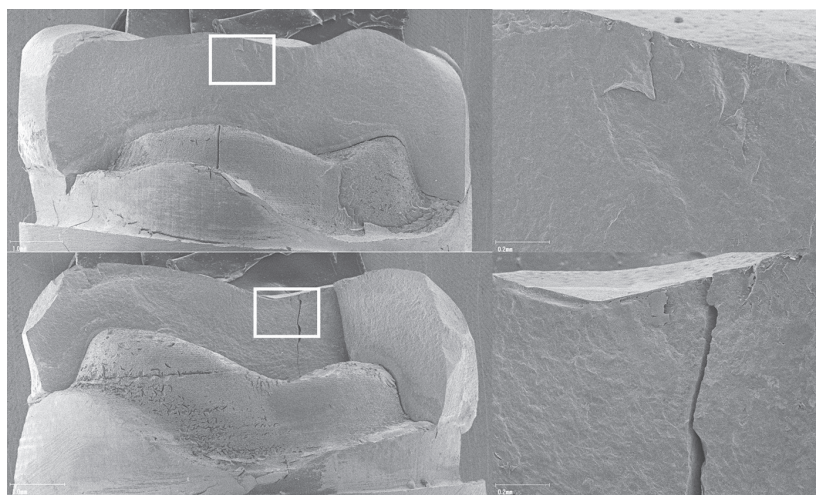

Fig. 7 Fractographic images of partial coverage crowns with finish line at $4 \mathrm{~mm}$ above the gingiva for Rosetta $\mathrm{BM}$ at $\times 20$ for $\mathrm{a}$ and $\mathrm{c}$, and $\times 100$ for $\mathrm{b}$ and $\mathrm{d}$ magnification for different specimens. 
had fewer cracks present than full coverage crowns, regardless of material type. This may be related to the restorative design, even though a significant difference in load at cracking and fracture was only observed for Rosetta BM. Fracture surfaces on the printed teeth for the crowns of IPS Empress CAD showed higher numbers of cracks vertically and horizontally that those of Rosetta BM. This is clearly related the weaker fracture resistance of the crowns of Rosetta BM.

\section{DISCUSSION}

The preservation of tooth substrates is paramount for minimal intervention dentistry ${ }^{15}$; thus fracture resistance of crowns with conservative restorative designs involving minimal tooth reduction compared with conventional approaches have been evaluated. A novel conservative preparation design for worn teeth is partial coverage crowns, which are no retentive bonded ceramic partial coverage crowns that cover the entire occlusal surface without extending the finish line to the gingival ${ }^{16)}$. Some laboratory studies have shown promising results with conservative ceramic partial coverage crowns, including occlusal veneers ${ }^{17,18}$. However, other systematic reviews of conservative partial coverage crowns using ceramic materials have reported survival rates after 5 to 10 years that are not promising ${ }^{19}$. Therefore, the longevity of crowns fabricated on conservative principles is still debatable, and additional investigations of conservative restorative designs in comparison to traditional full coverage crowns are desirable.

The present study investigated the fracture resistance of full and partial coverage crowns - partial coverage crowns finish line at $2 \mathrm{~mm}$ and $4 \mathrm{~mm}$ above the gingiva - fabricated with leucite reinforced ceramic restorative blocks from two different manufacturers. Previous studies revealed that the maximum occlusal force on the molar is $650.7-762.5 \mathrm{~N}$ for men and 553.0$652.5 \mathrm{~N}$ for women ${ }^{20,21)}$ Therefore, load at break of all tested crowns of IPS Empress CAD considerably exceeded the maximum occlusal force in the molars, regardless of the restorative design. However, a full coverage crown of Rosetta BM, with a load at break of $688.2 \mathrm{~N}$, may risk cracking after it has been in place for some time and subjected to fatigue, under excessive occlusal force. Thus, especially when using Rosetta BM for full coverage crowns for molars, clinicians should be cautious about the risk of fracture of the crown. On the other hand, the partial coverage crowns have a comfortable margin of safety over the reported bite forces.

The failure of some of the partial coverage crown samples during fatigue application was due to the failure of the resin abutment. In an actual tooth, the area between a partial coverage crown and the gingiva is encased in enamel, and thus has greater strength. However, the resin abutments were made entirely of material with properties similar to dentin, and were weaker than a tooth. This was seen in the failure of the resin teeth below the partial coverage crown in some cases. These failures are of no clinical interest, and provide no information about the strength of the restoration, and were therefore dropped from the analysis.

A previous study comparing the mechanical properties of IPS Empress CAD and Rosetta BM reported that the flexural strength and fracture toughness were 158.1 $\mathrm{MPa}$ and 1.28 $\mathrm{MPa}$ for IPS Empress CAD and 172.3 $\mathrm{MPa}$ and 1.30 $\mathrm{MPa}$ for Rosetta $\mathrm{BM}$, and both indicators showed significantly higher values for Rosetta BM than for IPS Empress ${ }^{14}$. These results are not easily reconciled with the present results, in which all the crowns using IPS Empress CAD showed significantly higher fracture resistance than those using Rosetta BM. Typically, the mechanical properties and strength of restorations show positive correlations ${ }^{22)}$. However, the results of this study do not follow this general trend; therefore additional investigations of the mechanical properties of Rosetta BM may be needed. The claim of the manufacturer of Rosetta BM that the material properties have been improved compared to previously marketed products is supported by other reported data on its mechanical properties, but not by the results of this study, which suggests that investigations of the fracture resistance of fabricated restorations are also needed. Further research is needed to identify the reasons for the disparity between the two kinds of results.

In this study, the fracture test of the crowns was conducted after fatigue loading using 1.0 million cycles with 100 N. Fracture tests that use only static loading are widely employed, but such tests can only show the fracture resistance of a crown immediately after bonding, and may not be suggestive of the longevity of the restoration ${ }^{23}$. A possible way to simulate the clinical situation in fracture testing of restorations is to apply fatigue loading before the static peak load test, as in this study. Tsujimoto et al. reported that an individual makes around 2,700 mastication cycles/day, or around 1.0 million cycles/year ${ }^{24}$. In addition, Skouridou et al. used 1.2 million cycles for fatigue testing to simulate 5 years clinical service ${ }^{23)}$. Furthermore, a loading force of $100 \mathrm{~N}$ was selected as an occlusal force during fatigue testing ${ }^{25}$. Therefore, these results represent fracture resistance after the application of clinically realistic fatigue.

The fracture resistance of full and partial coverage crowns of IPS Empress CAD was not influenced by the restorative design, unlike that of Rosetta BM. In addition, the full coverage crown of Rosetta BM was much weaker than the partial coverage crown. This result was surprising, as one might expect the larger full coverage crown to be much stronger than partial coverage crowns. This may be influenced by the differences in crystalline between IPS Empress CAD and Rosetta BM. However, another study has found that the fracture resistance of partial coverage crowns for a premolar using another type of reinforced ceramic block (IPS e.max CAD, Ivoclar Vivadent) was much higher than full coverage crowns ${ }^{18}$. As results like this are reported, the idea that one can fabricate a stronger restoration by removing more of the tooth material to make space for a larger crown may be 
changing.

The analysis of the fractography did seem to show some consistent differences between designs and materials. However, there was a great deal of variation between individual samples (see, for example, Fig. 7, where the upper sample is much cleaner than the lower sample), and there is no clear connection between the observed differences in fractography and the differences in load at break or peak load. Further investigations may shed more light on mechanisms, but we cannot say anything definitive based on this part of this experiment.

We hoped that measuring more values would allow us to shed light on such results. The time elapsed until fracture indicates how well the sample can respond to an increasing applied force and disperse it through the structure, thus avoiding sudden failure. Thus, the relationships between the time and the load at failure would, we hoped, shed more light on the mechanisms. However, in the event, there were very few significant differences between the times to fracture, because of the very large standard deviations, and so these results were not helpful in analyzing the process of failure. It is possible that experiments using a much larger number of samples might be able to find something useful from this measure.

Load at break and peak load represent the loads at which the material first cracks, and the load at which it fails completely, respectively. Both of these represent failures of the restoration, but in some cases a small crack might not be noticed by the patient, while a complete failure certainly would, and we thought that the differences in the relationships between these values might tell us something interesting about the process of failure in restorations of different designs. However, again partly due to large standard deviations, there were no clear differences in trends between the designs. A larger sample size might show something, but it is possible that there is simply no interesting relationship here.

Nevertheless, the main results of the experiment did show clear differences between materials and restoration designs. Overall, the first null hypothesis, that the two different materials would show no differences, was rejected, and the second and third null hypotheses, that different restorative designs would show no difference, were partially rejected.

\section{CONCLUSION}

The fracture resistance and fractographic analysis of $\mathrm{CAD} / \mathrm{CAM}$ leucite reinforced full and partial coverage crowns were influenced by the restorative design and the material type. Conservative partial coverage crowns with finish line at $2 \mathrm{~mm}$ and $4 \mathrm{~mm}$ above the gingiva showed comparable fracture resistance to full coverage crowns when fabricated with conventional leucite ceramic. In contrast, when fabricated with a newer leucite reinforced ceramic, full coverage crowns showed lower fracture resistance than partial crowns with finish line at $2 \mathrm{~mm}$ and $4 \mathrm{~mm}$ above the gingiva. These results suggest that partial coverage crowns should be seriously considered as a restoration option for worn teeth, but other clinical factors must also be taken into account.

\section{REFERENCES}

1) Tsujimoto A, Barkmeier WW, Fischer NG, Nojiri K, Nagura $\mathrm{Y}$, Takamizawa $\mathrm{T}$, et al. Wear of resin composites: Current insights into underlying mechanisms, evaluation methods and influential factors. Jpn Dent Sci Rev 2018; 54: 76-87.

2) Tsujimoto A, Jurado C, Villalobos-Tinoco J, Barkmeier W, Fischer N, Takamizawa T, et al. Wear resistance of indirect composite resins used for provisional restorations supported by implants. J Adv Prosthodont 2019; 11: 232-238.

3) Morimoto S, Rebello de Sampaio FB, Braga MM, Sesma N, Özcan M. Survival rate of resin and reramic inlays, onlays, and overlays: A systematic review and meta-analysis. J Dent Res 2016; 95: 985-994

4) Edelhoff D, Ahlers MO. Occlusal onlays as a modern treatment concept for the reconstruction of severely worn occlusal surfaces. Quintessence Int 2018; 49: 521-533.

5) Tsujimoto A, Barkmeier WW, Takamizawa T, Latta MA, Miyazaki M. Influence of thermal cycling on flexural properties and simulated wear of computer-aided design/ computer-aided manufacturing resin composites. Oper Dent 2017; 42: 101-110.

6) Sulaiman TA. Materials in digital dentistry -A review. J Esthet Restor Dent 2020; 32: 171-181.

7) Della Bona A, Nogueira AD, Pecho OE. Optical properties of CAD-CAM ceramic systems. J Dent 2014; 42: 1202-1209.

8) Alakkad L, Kostagianni A, Finkelman M, Maawadh A, Ali A, Papathanasiou A. Biaxial flexural strength of various CADCAM glass-ceramic materials. Am J Dent 2021; 34: 91-96.

9) Yoshida F, Tsujimoto A, Ishii R, Nojiri K, Takamizawa $\mathrm{T}$, Miyazaki M, et al. Influence of surface treatment of contaminated lithium disilicate and leucite glass ceramics on surface free energy and bond strength of universal adhesives. Dent Mater J 2015; 34: 855-862.

10) Fu L, Engqvist H, Xia W. Glass-ceramics in dentistry: A review. Materials (Basel) 2020; 13: 1049.

11) Keshvad A, Hooshmand T, Asefzadeh F, Khalilinejad F, Alihemmati M, Van Noort R. Marginal gap, internal fit, and fracture load of leucite-reinforced ceramic inlays fabricated by CEREC inLab and hot-pressed techniques. J Prosthodont 2011; 20: 535-540.

12) Guess PC, Strub JR, Steinhart N, Wolkewitz M, Stappert CF. All-ceramic partial coverage restorations - midterm results of a 5-year prospective clinical split mouth study. J Dent 2009; 37: 627-637.

13) Awada A, Nathanson D. Mechanical properties of resinceramic CAD/CAM restorative materials. J Prosthet Dent 2015; 114: 587-593.

14) Byeon SM, Song JJ. Mechanical properties and microstructure of the leucite-reinforced glass-ceramics for dental CAD/CAM. J Dent Hyg Sci 2018; 18: 42-49.

15) Jurado C, Watanabe H, Tinoco JV, Valenzuela HU, Perez GG, Tsujimoto A. A conservative approach to ceramic veneers: A case report. Oper Dent 2020; 45: 229-234.

16) Luciano M, Francesca Z, Michela S, Tommaso M, Massimo A. Lithium disilicate posterior overlays: Clinical and biomechanical features. Clin Oral Investig 2020; 24: 841848.

17) Politano G, Van Meerbeek B, Peumans M. Nonretentive bonded ceramic partial crowns: Concept and simplified protocol for long-lasting dental restorations. J Adhes Dent 2018; 20: 495-510.

18) Huang X, Zou L, Yao R, Wu S, Li Y. Effect of preparation 
design on the fracture behavior of ceramic occlusal veneers in maxillary premolars. J Dent 2020; 97: 103346.

19) Pieger S, Salman A, Bidra AS. Clinical outcomes of lithium disilicate single crowns and partial fixed dental prostheses: A systematic review. J Prosthet Dent 2014; 112: 22-30.

20) Sathyanarayana HP, Premkumar S, Manjula WS. Assessment of maximum voluntary bite force in adults with normal occlusion and different types of malocclusions. J Contemp Dent Pract 2012; 13: 534-538.

21) Padma S, Umesh S, Asokan S, Srinivas T. Bite force measurement based on fiber Bragg grating sensor. J Biomed Opt 2017; 22: 1-6.

22) Furtado de Mendonca A, Shahmoradi M, Gouvêa CVD, De Souza GM, Ellakwa A. Microstructural and mechanical characterization of CAD/CAM materials for monolithic dental restorations. J Prosthodont 2019; 28: e587-e594.

23) Skouridou N, Pollington S, Rosentritt M, Tsitrou E. Fracture strength of minimally prepared all-ceramic CEREC crowns after simulating 5 years of service. Dent Mater 2013; 29: e70e77.

24) Tsujimoto A, Barkmeier WW, Erickson RL, Fischer NG, Markham MD, Takamizawa T, et al. Shear fatigue strength of resin composite bonded to dentin at physiological frequency. Eur J Oral Sci 2018; 126: 316-325.

25) Yazigi C, Schneider H, Chaar MS, Rüger C, Haak R, Kern M. Effects of artificial aging and progression of cracks on thin occlusal veneers using SD-OCT. J Mech Behav Biomed Mater 2018; 88: 231-237. 